\title{
SOPHIE velocimetry of Kepler transit candidates: a joint photometric, spectroscopic and dynamical analysis of the Kepler-117 system
}

\author{
G. Bruno ${ }^{1, a}$, J.-M. Almenara ${ }^{1}$, S. C. C. Barros ${ }^{1}$, A. Santerne ${ }^{2}$, R. F. Díaz ${ }^{1,5}$, M. Deleuil ${ }^{1}$, C. Damiani ${ }^{1}$,
} A. S. Bonomo ${ }^{6}$, I. Boisse ${ }^{1}$, F. Bouchy ${ }^{1}$, G. Hébrard ${ }^{3,4}$, and G. Montagnier ${ }^{3,4}$

1 Aix Marseille Université, CNRS, LAM (Laboratoire d'Astrophysique de Marseille) UMR 7326, 13388, Marseille, France

2 Centro de Astrofisica, Universidade do Porto, Rua das Estrelas, 4150-762 Porto, Portugal

3 Observatoire de Haute Provence, 04670 Saint Michel l'Observatoire, France

4 Institut d'Astrophysique de Paris, UMR7095 CNRS, Université Pierre \& Marie Curie, 98bis boulevard Arago, 75014 Paris, France

5 Observatoire Astronomique de l'Université de Genève, 51 chemin des Maillettes, 1290 Versoix, Switzerland

6 INAF - Osservatorio Astrofisico di Torino, via Osservatorio 20, 10025 Pino Torinese, Italy

\begin{abstract}
We present the analysis of the multi-planetary system Kepler-117, which is part of our program of observations of Kepler planets. This system is composed of a $\sim 30 M_{\text {Earth }}$ planet in a $\sim 19$ days orbit and a $\sim 2 M_{\mathrm{J}}$ planet orbiting in $\sim 51$ days. Both the orbits have low eccentricity. The planets are not close to an exact low-order mean motion resonance, but exhibit significant transit timing variations (TTVs) nevertheless. We perform a combined Markov Chain Monte Carlo fit on all the available data: the Kepler photometry, the TTVs, the radial velocities we obtained with SOPHIE/OHP and the stellar parameters. The prime result is that the modelling of the TTVs allows to increase the precision on the system parameters which are not constrained by the radial velocities alone.
\end{abstract}

\section{Introduction}

Multiple planet systems offer insights on their dynamical history [1] and can show transit timing variations (TTVs; [2]). TTVs are a powerful tool for the detection of non-transiting planets, the determination of planetary masses (e.g. [3]) and the track of stellar activity (e.g. [4]). Unaccounted-for TTVs can cause an underestimation of the uncertainty on the stellar density derived from the photometry [5]. Kepler-117 has been observed in the context of our program of ground-based follow-up of Kepler planetary candidates (e.g. [6]). The planets Kepler-117 b and c have been validated by [7] with a confidence level of more than $99 \%$, after passing several tests for the identification of false positives.

\section{Data and methods}

We made use of the Kepler photometry, from quarter 1 to 3 (long cadence data, 29.4 minutes) and from 4 to 17 (short cadence, 58.5 seconds). The radial velocities (RVs) were obtained with SOPHIE/OHP between 2012 and 2013 (spectral resolution $\simeq$ 39000). The spectrum of the host star was obtained

a e-mail: giovanni.bruno@lam.fr

This is an Open Access article distributed under the terms of the Creative Commons Attribution License 4.0, which permits unrestricted use, distribution, and reproduction in any medium, provided the original work is properly cited. 

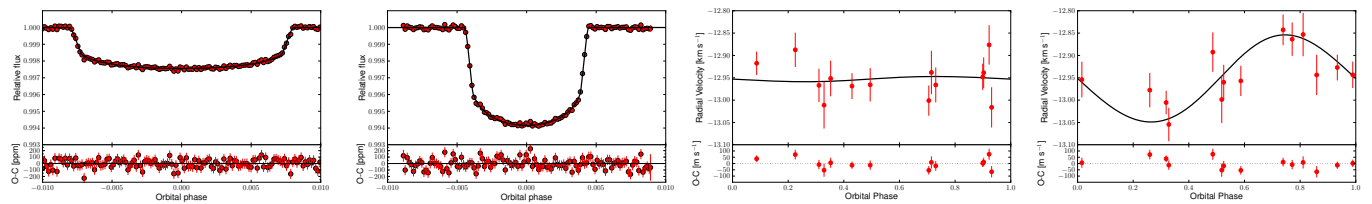

Fig. 1. From left to right: phase-folded plot of the best transit model of planet $b$ and c, over the short cadence data; the phase-folded RVs and the corresponding best model for planet $\mathrm{b}$ and $\mathrm{c}$. The lower panels show the residuals as observed minus calculated $(O-C)$ points. Reference: [11]

Table 1. Planetary and stellar parameters with their $68.3 \%$ central confidence intervals.

\begin{tabular}{lll}
\hline$T_{\text {eff }}[\mathrm{K}]$ & $6150 \pm 110$ & \\
Metallicity $[\mathrm{Fe} / \mathrm{H}]$ & $-0.04 \pm 0.10$ & \\
Derived $\log g$ & $4.10 \pm 0.02$ & \\
Stellar density $\rho_{\star}\left[\rho_{\odot}\right]$ & $0.29_{-0.02}^{+0.01}$ & \\
Stellar mass $\left[M_{\odot}\right]$ & $1.13_{-0.02}^{+0.13}$ & \\
Stellar radius $\left[R_{\odot}\right]$ & $1.61 \pm 0.05$ & \\
\hline Planet parameters & Kepler- $-117 b$ & Kepler- $117 \mathrm{c}$ \\
Orbital period $P[$ days $]$ & $18.80 \pm 7.5 \times 10^{-6}$ & $50.79 \pm 1.4 \times 10^{-5}$ \\
Orbital eccentricity $e$ & $0.05 \pm 0.01$ & $0.03 \pm 0.003$ \\
Planet mass $M_{p}\left[\mathrm{M}_{J}\right]$ & $0.09 \pm 0.03$ & $1.84 \pm 0.18$ \\
Planet radius $R_{p}\left[\mathrm{R}_{J}\right]$ & $0.72 \pm 0.02$ & $1.10 \pm 0.04$ \\
Planet density $\rho_{p}\left[\mathrm{~g} \mathrm{~cm}^{-3}\right]$ & $0.30 \pm 0.11$ & $1.74 \pm 0.18$ \\
\hline
\end{tabular}

from the RV spectra and analyzed with the VWA package (e.g. [8]) to derive the stellar parameters. The transit times were fitted with a procedure similar to the one discussed in [4].

The photometry, the RVs and the stellar parameters were jointly fitted with the Bayesian-oriented PASTIS software [9]. The parameters posteriors have been sampled with a MCMC algorithm. The TTVs modelling has been included, as well, using the orbital dynamics software mercury [10].

\section{Results}

The system exhibits significant TTVs even if the period ratio is not close to an exact low-order mean motion resonance. The inclusion of the TTVs in the combined fit allows to fit parameters that are poorly constrained with the RVs only (fig.1, [11]). The mass of planet b, whose RV amplitude is close to the limit of efficiency of SOPHIE for faint magnitudes, is precisely determined . Finally, with a mass ratio close to 20 and similar radii, we found the two planets to have considerably different densities.

\section{References}

1. Batygin, K. \& Morbidelli, A., AJ, 145, 1 (2013)

2. Agol, E., Steffen, J., Sari, R., \& Clarkson, W., MNRAS, 359, 567 (2005)

3. Holman, M. J., Fabrycky, D. C., Ragozzine, D., et al., Science, 330, 51 (2010)

4. Barros, S. C. C., Boué, G., Gibson, N. P., et al., MNRAS, 430, 3032 (2013)

5. Kipping, D. M., MNRAS, 440, 2164 (2014)

6. Bouchy, F., Bonomo, A. S., Santerne, A., et al., A\&A, 533, A83 (2011)

7. Rowe, J. F., Bryson, S. T., Marcy, G. W., et al., ApJ, 784, 45 (2014)

8. Bruntt, H., Bedding, T. R., Quirion, P.-O., et al., MNRAS, 405, 1907 (2010)

9. Díaz, R. F., Almenara, J. M., Santerne, A., et al., MNRAS, 441, 983 (2014)

10. Chambers, J. E., MNRAS, 304, 793 (1999)

11. Bruno, G., Almenara, J.-M., Barros, S. C. C., et al. (submitted) 